\title{
Rayleigh surface waves propagating in (111) Si substrate decorated with Ni phononic nanostructure
}

\author{
B. Graczykowski ${ }^{1}$, S. Mielcarek ${ }^{1}$, A. Trzaskowska ${ }^{1}$, P. Patoka $^{2}$, M. Giersig ${ }^{2}$ \\ ${ }^{1}$ Faculty of Physics, Adam Mickiewicz University, Umultowska 85, 61-614 Poznań, Poland \\ ${ }^{2}$ Freie Universität Berlin, Institut für Experimentalphysik, Arnimallee 14, 14195 Berlin, Germany
}

\begin{abstract}
The paper reports results of the Surface Brillouin Light Scattering at the silicon (111) surface loaded with a periodic 2D nickel nanostructure. Measurements were made for samples loaded with nanostructures of different period (different size) but of the same height. The relation between the nanostructure size and the velocity of surface Rayleigh waves was proved to be nonlinear. Anisotropy of the surface Rayleigh wave velocity was compared with the results of theoretical modelling based on the Finite Element Method.
\end{abstract}

\section{INTRODUCTION}

Deposition of a 2D periodic structure on a substrate having different elastic properties should bring about changes in the velocity of the Rayleigh Surface Waves (RSW) propagating in the substrate [1-5]. These waves can be observed in the hypersonic range by the method of Surface Brillouin Light Scattering (SBLS). The paper reports measurements of the velocity of RSW in silicon (111) decorated with a nickel nanostructure, for different distances between the nickel islands but at their constant height. The nickel nanostructure was produced by nanosphere lithography which permitted getting islands of almost triangular bases arranged into a honeycomb structure. Theoretical modelling was performed by the Finite Element Method (FEM) (COMSOL Multiphysics) and its results were confronted with the experimental data.

\section{MATERIALS AND METHODS}

The samples used in the experiment were made by our partner at the Freie Universität Berlin. Detail description of their production can be found in $[6,7]$. The samples differed in the size of the mask balls whose diameters were 496, 1040 or $1710 \mathrm{~nm}$. The height of the structure deposited was the same and equal to $25 \mathrm{~nm}$. Modification of the mask by changes in the ball diameter implies changes in distances between the islands and in their sizes. The orientation of the 2D nanostructure with respect to the crystallographic axes of the support is given in Fig. $3 \mathrm{~b}$ (insert). It should be noted that the ratio of the area occupied by the deposited nickel islands $S_{N i}$ to the area of unloaded silicon surface $S_{S i}$ is independent of the ball size. A good approximation is that:

$$
S_{N i} / S_{S i}=(2 \sqrt{3}-\pi) / \pi \cong 0.1, \quad S_{N i} /\left(S_{S i}+S_{N i}\right)=\pi / 2 \sqrt{3} \cong 0.91 .
$$

In view of the above, the question is what is the effect of the loading which is a nanostructure of the same weight but of different size on the Rayleigh surface wave of the silicon support.

International Congress on Ultrasonics

AIP Conf. Proc. 1433, 263-266 (2012); doi: 10.1063/1.3703185

(C) 2012 American Institute of Physics 978-0-7354-1019-0/\$30.00 


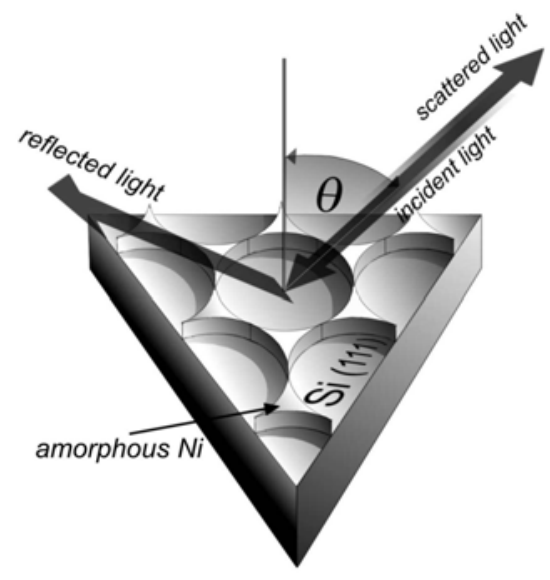

Fig 1. A schematic presentation of a fragment of the sample showing the SBLS measuring geometry.

The Rayleigh-type surface waves propagating in silicon (111) substrate covered by nickel nanostructure were investigated using the piezoelectrically scanned $(2 \times 3)$ pass tandem Fabry-Perot interferometer (JRS Scientific Instruments). The source of incident light was a Spectra-Physics laser operating at $\lambda=532 \mathrm{~nm}(200 \mathrm{~mW})$. Detailed description of the spectrometer can be found in [8]. If the angle of incidence is equal to the scattering angle denoted as $\theta$ (Fig.1), (the so-called backscattering geometry) the phase velocity $v_{R}$ of RSW can be calculated from the equation [8]:

$$
\Delta \omega_{B}=\frac{2 v_{R} \sin \theta}{\lambda} .
$$

where $\Delta \omega_{B}$ is the Brillouin shift, $\lambda$ the laser wavelength.

\section{RESULTS AND DISCUSSION}

Velocities of RSW measured for different $\theta$ angle suggest a linear relationship between $\Delta \omega_{B}$ and $2 \sin \theta$ (and hence on the wavenumber) in contrast to the results obtained for a uniform nickel layer (25 nm) supported on the same silicon substrate (see Fig. 2a). On the basis of the relation between $\Delta \omega_{B}$ and $2 \sin \theta$ the phase velocity of RSW was determined in the directions [110] and [11 $]$. The results for different size ball masks are presented in Fig. 2b. The are compared with the range of RSW velocity determined for the silicon support loaded with a uniform nickel layer of the same load weight per an area unit. The changes in RSW velocity caused by the nanostructure are greater than those attributed to a uniform nickel layer and the magnitude of the change depends on the size of the nanostructure; with increasing size of the island base (diameter of mask balls) the RSW velocity decreases. Fig. $2 \mathrm{~b}$ presents the fit of RSW as a function of the mask ball diameter by an exponential decay curve.

The anisotropy of RSW velocity for the sample obtained with the mask ball diameter $d=496 \mathrm{~nm}$ for $\theta=30^{\circ}$ was compared with the anisotropy obtained by simulation using the Finite Element Method (FEM). Moreover, the anisotropy obtained for the sample loaded with a nanostructure was compared with that obtained for pure silicon surface (111). As follows from Fig. 3a, the loading with nanostructure results in discontinuities and gaps in the anisotropy of RSW phase velocity. Their appearance can be interpreted as an impact of the periodic structure on SRW of phononic character. The appearance of such discontinuities is in agreement with the FEM modelling results (Fig.3b). In the range of theta angles studied, the results of the experiment as well as those of the FEM simulation of RSW phase velocity are not fully symmetrical, in contrast to the results obtained for pure silicon surface. It is a consequence of a small shift ( $\Delta \alpha \cong 2^{\circ}$ taken into account in the simulations) in the nanostructure orientation with respect to the orientation of the silicon substrate. 

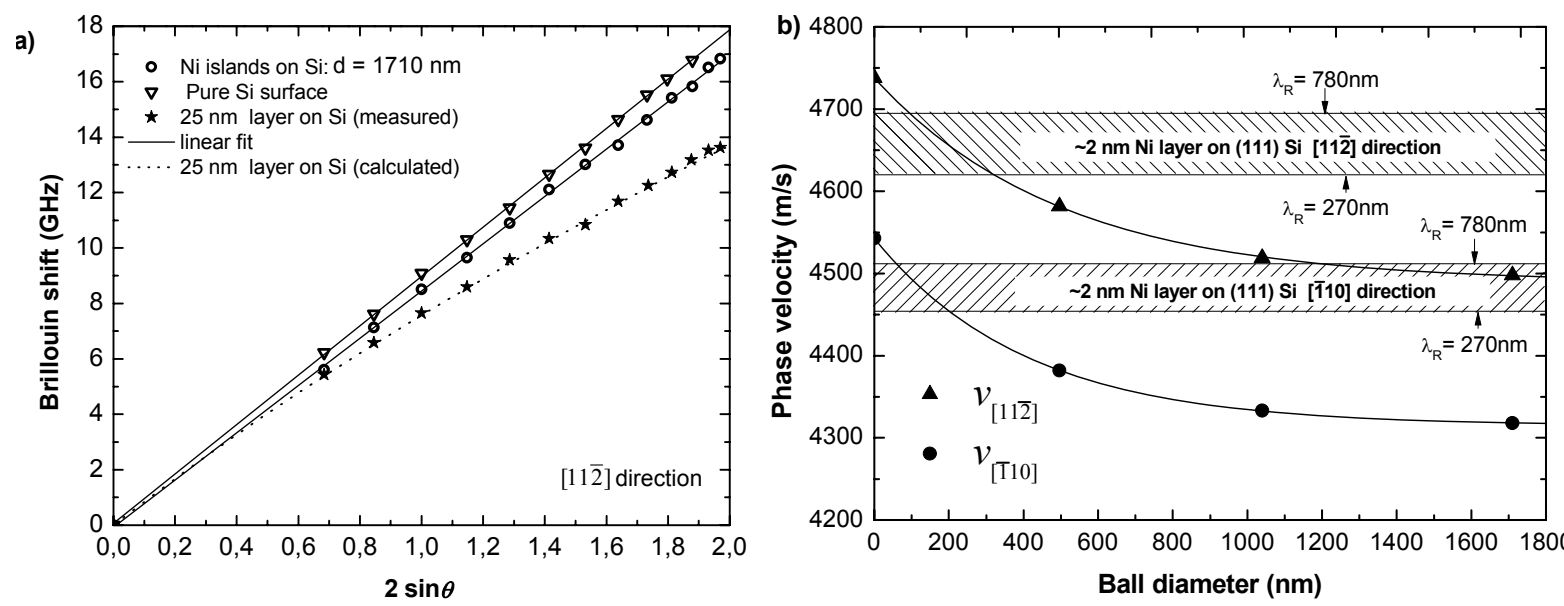

Fig. 2. a) Brillouin shift versus $2 \sin \theta$ for pure Si surface, $25 \mathrm{~nm} \mathrm{Ni}$ layer on $\mathrm{Si}$ and for $\mathrm{Si}$ decorated with nanostructure, b) RSW phase velocity of RSW versus ball diameter. The areas marked by skew lines represent results for $\sim 2 \mathrm{~nm}$ thick nickel layer on (111) $\mathrm{Si}$ - which correspond to the range of RSW wavelengths approximately from $780 \mathrm{~nm}$ to $270 \mathrm{~nm}$.
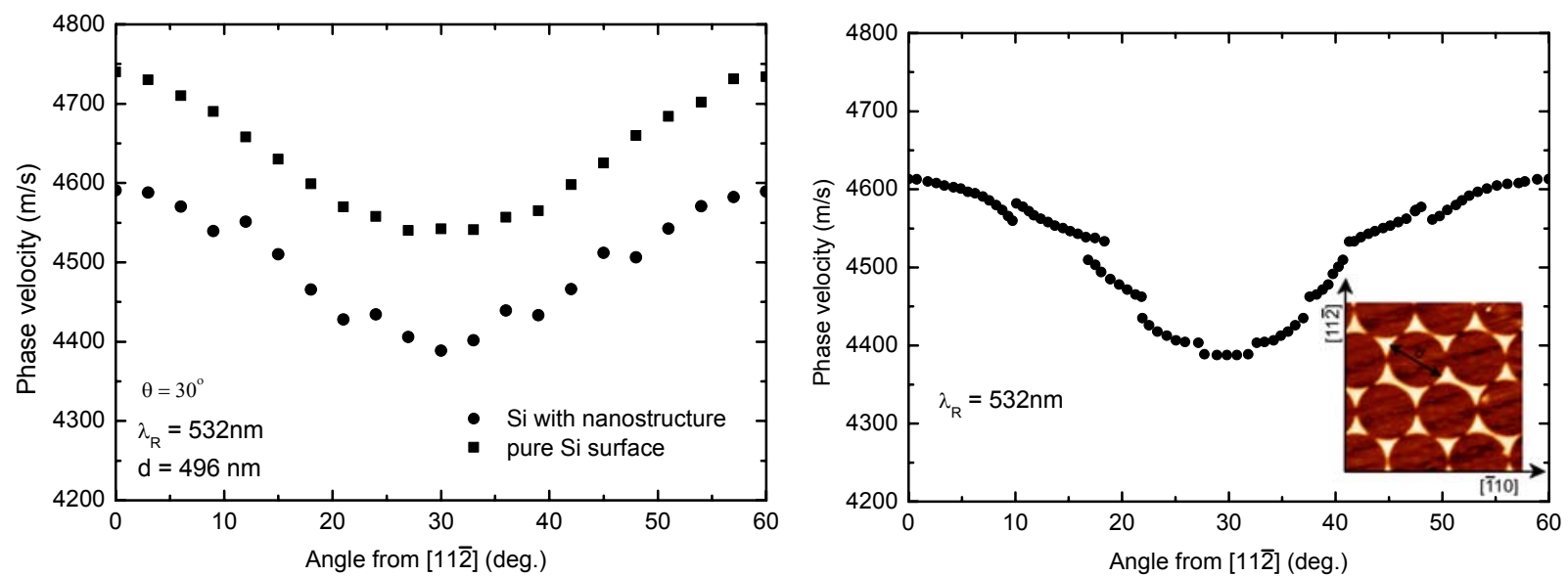

Fig. 3. a) Anisotropy of RSW velocity for (111) in silicon and (111) in silicon loaded with a nanostructure,

b) Results of FEM based simulation of RSW velocity anisotropy.

Results of the FEM based simulation for a few lengths of RSW are shown in Fig. 4 in the form of three-dimensional map of relative deformation of an arbitrary elementary cell (see Fig. 4) and for SRW of a few lengths. With decreasing RSW length the disturbance in its shape gets enhanced and concerns not only the substrate surface but also its bulk. The RSW velocities obtained from FEM based simulations were found by searching for the undamped eigenvalues of vibrations at the specific boundary conditions of elementary cell (periodic boundary conditions) for a present length of RSW. Only these solutions that corresponded to the maximum concentration of the deformation energy in the vicinity of the free surface were accepted. 


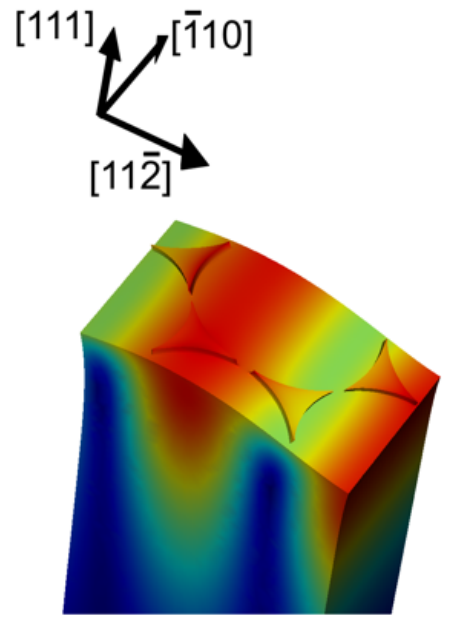

$\lambda_{R}=1150 \mathrm{~nm}$

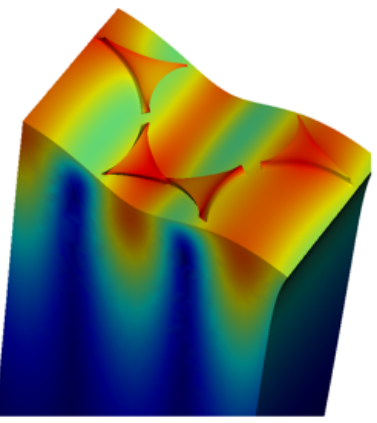

$\lambda_{R}=750 \mathrm{~nm}$

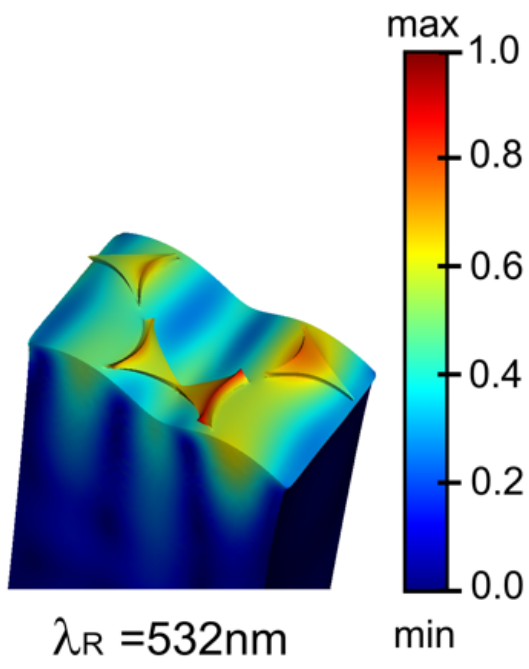

Fig. 4. Results of FEM based simulations obtained for a few length of RSW.

\section{CONCLUSIONS}

Surface Brillouin phonon scattering was applied to study the effect of a nickel nanostructure deposited on the silicon (111) surface on the surface Rayleigh waves velocity. With increasing size of the nanostructure (mask ball diameter) the surface Rayleigh wave velocity decreases, in the range accessible for SBLS. The change is greater in magnitude than that caused by loading the silicon surface with a uniform nickel layer of the same mass per unit area. Comparison of the experimental data with the results of FEM modelling has confirmed the impact of the nanostructure studied on the RSW velocity anisotropy.

\section{ACKNOWLEDGEMENTS}

This work has been partially supported by the Grant No. N202 230637 from the Polish Ministry of Science and Higher Education.

\section{REFERENCES}

[1] R.M. Hornreich, M. Kugler, S. Shtrikman, C. J. Phys. I France 7, 509-519 (1997).

[2] M. Sigalas, N. Garcia, J. Appl. Phys. 87, 3122-3125 (2000).

[3] D. Zhao, Z.Y. Liu, C.Y. Qiu, Z.J. He, F.Y. Cai, M.Z. Ke, Phys. Rev. B 76,144301 (2007).

[4] R. Sainidou, B. Djafari-Rouhani, J. Vasseur, Phys. Rev. B 77, 094304 (2008).

[5] F.R. Montero de Espinoza, E. Jimenez, M. Torres, Phys. Rev. Lett. 80, 1208 (1998).

[6] J. Rybczynski, U. Ebels and M. Giersig, Colloids and Surfaces A: Physicochem. Eng. Aspects 219, 1-6 (2003).

[7] A. Kosiorek, W. Kandulski, P. Chudzinski, K. Kempa and M. Giersig, Nano Letters 4, 1359-1363 (2004).

[8] J.R. Sandercock, Trends in Brillouin Scattering in Topics in Applied Physics 51 (Springer, Berlin, 1982). 
Copyright of AIP Conference Proceedings is the property of American Institute of Physics and its content may not be copied or emailed to multiple sites or posted to a listserv without the copyright holder's express written permission. However, users may print, download, or email articles for individual use. 